\title{
Role of generosity and forgiveness: Return to a cooperative society
}

\author{
Hye Jin Park, ${ }^{1,2}$ Beom Jun Kim, ${ }^{1, *}$ and Hyeong-Chai Jeong ${ }^{3, \dagger}$ \\ ${ }^{1}$ Department of Physics, Sungkyunkwan University, Suwon 16419, Korea \\ ${ }^{2}$ Department of Evolutionary Theory, Max Planck Institute for Evolutionary Biology, 24306 Plön, Germany \\ ${ }^{3}$ Department of Physics and Astronomy, Sejong University, Seoul 05006, Korea
}

(Received 13 December 2016; published 17 April 2017)

\begin{abstract}
One's reputation in human society depends on what and how one did in the past. If the reputation of a counterpart is too bad, we often avoid interacting with the individual. We introduce a selective cooperator called the goodie, who participates in the prisoner's dilemma game dependent on the opponent's reputation, and study its role in forming a cooperative society. We observe enhanced cooperation when goodies have a small but nonzero probability of playing the game with an individual who defected in previous rounds. Our finding implies that even this small generosity of goodies can provide defectors chances of encountering the better world of cooperation, encouraging them to escape from their isolated world of selfishness.
\end{abstract}

DOI: 10.1103/PhysRevE.95.042314

\section{INTRODUCTION}

What is best for a myopic individual may not be the best for a long-lasting society. Suppose that two persons A and $\mathrm{B}$ trade their goods $\alpha$ and $\beta$ for their own benefits: A needs $\beta$ and B needs $\alpha$. One can maximize one's profit by giving nothing or inappropriate goods to one's counterpart in return for getting his or her wanted goods. Whether or not the opponent cheats, one is always better off cheating the trade partner. Consequently, both individuals, if they are rational, decide to cheat, although the total profit of the two is maximum when both of them cooperate and remain loyal. Such a situation, in which the individual and social benefits are in conflict with each other is called a social dilemma, with the prisoner's dilemma game (PDG) the most popular example $[1,2]$. In the conventional PDG, each player can cooperate or defect. Defection always gives a better profit irrespective of what the opponent does. Rationality of all individuals is assumed in the sense that all players try to maximize their own profits without considering the total profit of the society. The direct outcome from the setting of the conventional PDG is that all the players defect in the end, which is a Nash equilibrium of the conventional PDG.

From the viewpoint of natural selection, the behavior that gives the lower payoff measured by the number of offspring must eventually die out. Accordingly, if a cooperating individual gets a lower payoff than defecting individuals, defection must flourish in the end in this brutal world of Darwinian selection: Cooperation must be a rare thing in reality. Nevertheless, cooperative behaviors are abundantly observed in human societies and natural worlds. For this reason, extensive research has been carried out to find a mechanism that makes cooperative behavior evolutionary stable $[3,4]$.

Here, we focus on the indirect reciprocity [5-11] which explains the emergence of the cooperation in the following context: People tend to cooperate with a person who has a good reputation and thus is expected to cooperate back. Indirect reciprocity has been found to play an important

\footnotetext{
*Corresponding author: beomjun@skku.edu

${ }^{\dagger}$ Corresponding author: hcj@sejong.ac.kr
}

role in "third party altruism" [12] among strangers. Due to the fast development of Internet technology, more and more buyers get product information online and a huge volume of products is traded based on e-commerce. In a typical online shopping system, purchases of products are made between almost-anonymous buyers and sellers who have never met before. We believe that the success of such online trading hinges on the existence of a reliable and trustful reputation system. In this respect, indirect reciprocity must be the key factor for maintaining the online marketplace, on which experimental investigations have been performed $[13,14]$.

Previously, optional interactions based on indirect reciprocity have been examined [8] in terms of establishing a reputation. Reputation value has been treated as a binary variable, and thus defectors are not permitted to play games when the defector's reputation is established. In the present work, on the other hand, we more generally introduce nonbinary reputation values with a memory horizon $\tau$ and participation probability function based on the reputation value. Whereas previous works have focused on establishing a reputation, we concentrate on participation strategy with public reputation values. The main theme of the paper is to study the role of preferential participation in the survival of cooperators. We formulate the evolution of the population strategies and find the role of generosity and forgiveness for the evolution of cooperation. For $\tau=1$, if we lower the participation barrier for defectors, we observe that such generosity enhances cooperation in a society. For $\tau>1$, the preferential participation rule plays an important role in the evolution of cooperation by naturally introducing forgiveness in the sense that a one-time defection can be forgiven in view of a long sequence of cooperation in the past. The remainder is organized as follows: we introduce our model in Sec. II in a general context, and its mean-field formulation is described in Sec. III. Our mean-field results are reported in Sec. IV, and in Sec. V we summarize our results and interpret our conclusion in the setting of social cooperation.

\section{MODEL}

People repeatedly meet and interact with each other in a society. Depending on what a player and opponents do, the player gets payoffs from interaction. Accordingly, people tend 
to avoid playing with low bad-reputation partners. Defecting cheaters can get a large payoff the first time, but the cheaters have a hard time playing the game in the next round since they are now tagged as cheaters due to their bad reputation: Defectors can cheat once but not twice, because selective cooperators avoid playing the game with them. Hence, we focus on the option to refuse the game based on the partner's reputation.

We study the evolution of the stochastic optional PDG [15-17] in a complete graph. A player can choose the "refusal" option to opt out of the game. Each player proposes a game to his or her neighbors with a certain probability which depends on the neighbors' reputation. Two players actually play the game only when they propose the game mutually. For the sake of simplicity, we consider only two types of players, cheaters and goodies. Cheaters are indiscriminative defectors. They are not concerned about others' reputations and propose games for their neighbors at a given constant probability $\eta \in[0,1]$. Cheaters always defect once they play games. Goodies, on the other hand, are discriminative cooperators. When they play games they always cooperate, but they are picky in choosing games in which to participate. Goodies propose games to their neighbors depending on their reputations (see below for details).

In general, the reputation of a player reflects what the person has done in the past. It is fully determined by the strategy history of the player. We denote the strategy of the $i$ th player at time $t$ as $s_{i}(t)$. It is 1 and 0 for the goodie and the cheater strategies, respectively. The more frequently they have played goodie in the past, the better will be their current reputation. Reputation value is defined as the fraction of games in which the player has chosen the goodie strategy in the past. We also incorporate the fact that no one remembers the past for eternity and introduce the memory horizon $\tau$, which is a time cutoff in the calculation of the reputation value. For $t>0$, the reputation value $r_{i}(t)$ of player $i$ at time $t$ is defined as

$$
r_{i}(t)=\frac{1}{\tilde{\tau}} \sum_{t^{\prime}=t-\tilde{\tau}}^{t-1} s_{i}\left(t^{\prime}\right),
$$

where $\tilde{\tau}=\min (\tau, t)$ is the minimum of $\tau$ and $t$. At time $t=0$, when the first game begins, there is no reputation value to consult and thus we assign $r_{i}(t=0)=1 / 2$ for all players. We also assume that the values of reputations are faithful and accessible to all players.

As mentioned above, the game proposal probability of a player depends on the player's strategy and the reputation of the partner. We denote the probability $p_{s}(r)$ of a player with strategy $s$ to propose a game to a neighbor with reputation $r$ as

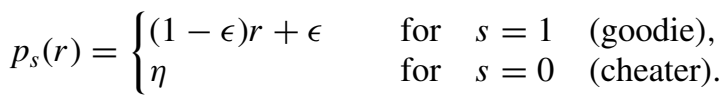

In words, $p_{0}(r)=\eta$ means that a cheater $(s=0)$ does not discriminate his or her counterpart, while $p_{1}(r)=(1-\epsilon) r+$ $\epsilon$ implies that a goodie $(s=1)$ is picky and proposes the game depending on the reputation of the partner.

The reputation value $r(t)$ defined by Eq. (1) is used when a goodie decides whether or not to propose a game to its potential partner. Since $p_{1}(r)$ is an increasing function of $r$, a goodie has a higher probability of playing the game with someone with a better reputation. The "generosity" parameter $\epsilon$ plays an important role for $\tau=1$ in our model. If it is $0(\epsilon=0)$, zero-reputation players (with $r_{i}=0$ ) cannot play games with goodies regardless of their current strategy. Hence, they will play only with cheaters if $\epsilon=0$, while a nonzero $\epsilon$ provides the route for the zero-reputation members to play games with goodies.

From our preferential participation rule in Eq. (2), forgiveness naturally emerges for $\tau>1$. Forgiveness is a reward response to one's past cooperative behavior. Therefore, we can interpret nonzero participation probabilities for nonzero reputation partners as forgiveness. In contrast to generosity, forgiveness does not work for null reputation partners. However, similarly to generosity, forgiveness also provides chances for cheaters to play games with goodies by remembering their past cooperative behaviors.

Once a pair plays the game (after mutual proposals), each player gets the payoff of the PDG. We consider a simplified version of the PDG, "donation" PDG, in which payoffs are expressed in terms of the cost $c$ and the benefit $b$ of the cooperation [6]. Cooperators pay the cost $c$ and the counterpart gets benefit $b$. Defectors do not pay the cost and no benefit occurs. If we use the notation $\left|\mathbf{s}_{i}\right\rangle=\left(\begin{array}{l}1 \\ 0\end{array}\right)$ for a cooperator and $\left|\mathbf{s}_{i}\right\rangle=\left(\begin{array}{c}0 \\ 1\end{array}\right)$ for a defector, the total payoff of the $i$ th agent is written as

$$
E_{i}(t)=\sum_{j \in \Omega_{i}}\left\langle\mathbf{s}_{i}(t)|\mathbf{M}| \mathbf{s}_{j}(t)\right\rangle,
$$

where $\Omega_{i}$ is the set of all neighbors who proposed the game mutually with $i$, and the payoff matrix is given by

$$
\mathbf{M}=\left(\begin{array}{cc}
b-c & -c \\
b & 0
\end{array}\right) .
$$

From now on, we fix the parameter $b=1$ without loss of generality and vary $c$ within the PDG constraint. Since the reward $R$, the temptation $T$, the sucker's payoff $S$, and the punishment $P$ in the conventional PDG correspond to $R=1-$ $c, T=1, S=-c$, and $P=0$ in our setting, we impose $0<$ $c<1$ for the PDG constraint $T>R>P>S$ (with $2 R>$ $T+S$ ). A player updates his or her strategy by mimicking a successful one. Before updating the player's reputation, his or her strategy is known only to directly interacting other individuals. Hence, adoption of a more successful strategy occurs only via direct interaction. In other words, we assume that the reputation is public information, but a player's current strategy is local information before it is reflected in his or her reputation.

The games are played as follows: (i) Each player proposes games to his or her neighbors at the probability given in Eq. (2). If two players mutually propose, they play the game. Otherwise, they do not play the game. Since player $i$ proposes a game to player $j$ at probability $p_{s_{i}}\left(r_{j}\right)$ and player $j$ does the same to player $i$ at probability $p_{s_{j}}\left(r_{i}\right)$, the two play the game with joint probability,

$$
p_{i j}=p_{s_{i}}\left(r_{j}\right) p_{s_{j}}\left(r_{i}\right)
$$

(ii) Each player gets payoffs from the games. The total payoff that player $i$ earns at time $t$ is $E_{i}(t)$ in Eq. (3). (iii) Strategies 
are updated synchronously. Every player randomly picks one of his or her game partners. If the neighbor's total payoff is higher than the player's own, the player follows the strategy of the chosen neighbor. Error (or mutation) can occur during the updating process at the mutation probability $\mu$ : Each player adopts the successful strategy of a chosen neighbor at probability $1-\mu$ and chooses another strategy at probability $\mu$. (iv) Reputations in Eq. (1) are also synchronously updated for the next round of iteration.

To summarize our model, there are five control parameters: The memory horizon $\tau$ controls how distant a past a player remembers for the reputation. Two parameters, $\eta$ and $\epsilon$, in Eq. (2) determine the proposal probabilities for cheaters and goodies, respectively, and we call the latter parameter $\epsilon$ generosity henceforth. We fix $b=1$ and use the cooperation $\cos t c$ in the payoff matrix as another parameter in the present paper. We also control the mutation probability $\mu$ that appears in the strategy update.

\section{EVOLUTIONARY DYNAMICS}

We consider our optional PDG model in a complete graph. Each player's behavior is described by what the person did in the past and does today, and we use the term state to denote each player's strategy history profile within the memory horizon $\tau$ and current strategy.

The state $S_{i}(t)$ of player $i$ at time $t$ is defined as

$$
S_{i}(t)=\sum_{k=0}^{\tilde{\tau}} 2^{k} s_{i}(t-k)
$$

with $\tilde{\tau}=\min (t, \tau)$. It can have $\tilde{N}=2^{\tilde{\tau}+1}$ different integer values, $S=0,1,2, \ldots, \tilde{N}-1$. We set $s_{i}(k)=0$ for all $k<0$ so that the state of Eq. (6) can be written as

$$
S_{i}(t)=\sum_{k=0}^{\tau} 2^{k} s_{i}(t-k)
$$

for $t<\tau$ as well as $t \geqslant \tau$. If we read $S_{i}$ as a binary number, it becomes $s_{i}(t-\tau) s_{i}(t-\tau+1) \ldots s_{i}(t-1) s_{i}(t)$. For example, when $\tau=1$, we have four possible states, 00 , 01,10 , and 11 , for $t \geqslant 1$ and two possible states, 00 and 01 , for $t=0$. For convenience, we also use " $\mathrm{H}$ " and "G" (for 0 and 1, respectively) to represent cheater and goodie, respectively. (To denote a cheater we use $\mathrm{H}$, which is the second letter in it instead of the first letter, $\mathrm{C}$, because the latter has been widely used to denote a cooperator). For instance, HG (01) means that the player was a cheater one time step ago and becomes a goodie now. If two players, $i$ and $j$, are in the same state $\left[S_{i}(t)=S_{j}(t)\right]$, they have the same reputation $\left[r_{i}(t)=r_{j}(t)\right]$ and (current) strategy $\left[s_{i}(t)=s_{j}(t)\right]$. Therefore, in a complete graph, total payoffs of the players in the same state are the same, and the population dynamics is fully determined by the frequency profile of states. We define the $N=2^{\tau+1}$-dimensional frequency vector $\mathbf{x}=\left(x_{0}, x_{1}, x_{2}, \ldots, x_{S}, \ldots, x_{N-1}\right)$, where the $S$ th component $x_{S}$ is the frequency of state $S$. For $t<\tau$, we set $x_{i}=0$ for $i>\tilde{N}$. In the same spirit, we define the $N$-dimensional total payoff vector $\mathbf{E}=\left(E_{0}, E_{1}, E_{2}, \ldots, E_{N-1}\right)$, where the $S$ th component $E_{S}$ is the total payoff of an individual in state $S$.
An individual in state $S$ has the reputation value $r_{S}=$ $\frac{1}{\tilde{\tau}} \sum_{k=1}^{\tilde{\tau}} s(t-k)$, which corresponds to the fraction of goodie strategies in the past $\tilde{\tau}$ time steps [see Eq. (1)]. From Eq. (2), we get the probability that a player in state $S$ proposes a game to a player in state $S^{\prime}$,

$$
p_{s(t)}\left(r_{S^{\prime}}\right)=\left[(1-\epsilon) r_{S^{\prime}}+\epsilon\right] s(t)+\eta[1-s(t)] .
$$

Since the game is played only for mutual proposals, the probability $p_{S S^{\prime}}$ that an individual in $S$ plays the game with an individual in $S^{\prime}$ is given by

$$
\begin{aligned}
p_{S S^{\prime}}= & p_{s(t)}\left(r_{S^{\prime}}\right) p_{s^{\prime}(t)}\left(r_{S}\right) \\
= & \left(\left[(1-\epsilon) r_{S^{\prime}}+\epsilon\right] s(t)+\eta[1-s(t)]\right) \\
& \times\left(\left[(1-\epsilon) r_{S}+\epsilon\right] s^{\prime}(t)+\eta\left[1-s^{\prime}(t)\right]\right) .
\end{aligned}
$$

When $S$ and $S^{\prime}$ (their strategies at $t$ are $s$ and $s^{\prime}$, respectively) play the PDG, the payoff that $S$ gets from $S^{\prime}$ is given by

$$
a_{S S^{\prime}}=b s^{\prime}-c s .
$$

Hence, the total payoff $E_{S}(t)$ that an individual in state $S$ earns at time $t$ is given by

$$
E_{S}(t)=\sum_{S^{\prime}=0}^{N-1} a_{S S^{\prime}} p_{S S^{\prime}} x_{S^{\prime}} .
$$

After all games are played at time $t$ for the whole population, players change their strategies as described in step (iii) in Sec. II: Player $i$ randomly selects one among its game partners (say, player $j$ ) and adopts the partner's strategy if $E_{j}>E_{i}$ (with probability $1-\mu$ and the opposite with probability $\mu$ ). We update strategies synchronously. The strategy $s_{i}$ of player $i$ at $t+1$ is given by $s_{i}(t+1)=1-s_{i}(t)$ if $E_{j}(t)>E_{i}(t)$ and $s_{j}(t) \neq s_{i}(t)$ (unless mutation occurs). Otherwise, $s_{i}(t+1)=$ $s_{i}(t)$. Now, state $S_{i}$ of player $i$ at $t+1$ is formally given by

$$
S_{i}(t+1)=2\left[S_{i}(t)-2^{\tau} s_{i}(t-\tau)\right]+s_{i}(t+1) .
$$

The meaning of Eq. (12) is easily understood in the binary representation of $S$. The updated strategy $s(t+1)$ becomes a new bit at the last binary digit ( $s=0$ or 1 depending on its strategy at $t+1)$ of $S(t+1)$. Other digits are simply obtained from the bits of $S(t)$ by just shifting each bit to the left, because the $k$ th past at time $t$ has become the $(k+1)$ th past at time $t+1$. For $t \geqslant \tau$, the leftmost bit of $S(t)$ is simply dropped in $S(t+1)$. Consequently, for a given state $S(t)$, only two states are possible for $S(t+1)$. In order to exclude forbidden transitions from $S$ to $S^{\prime}$, it is convenient to define

$$
\Lambda_{S S^{\prime}} \equiv \prod_{k=0}^{\tau-1} \delta_{\lfloor S\rfloor_{k}\left\lfloor S^{\prime}\right\rfloor_{k+1},}
$$

where

$$
\lfloor S\rfloor_{k} \equiv\left(\frac{S}{2^{k}}\right) \quad \bmod 2
$$

is the $(k+1)$ th binary bit of $S$. Then $\Lambda_{S S^{\prime}}=0$ for all $N-2$ forbidden transitions.

Since the change of a player's strategy is caused by a neighbor who has a higher total payoff $\left(E_{S^{\prime}}>E_{S}\right)$ with a different (current) strategy $\left(s^{\prime} \neq s\right)$, the probability $w_{S}$ of a 
player's changing his or her current strategy $s$ is given by

$$
w_{S}=\frac{1}{\sum_{S^{\prime}} p_{S S^{\prime}} x_{S^{\prime}}} \sum_{S^{\prime}} p_{S S^{\prime}} x_{S^{\prime}}\left(1-\delta_{S S^{\prime}}\right) \Theta\left(E_{S^{\prime}}-E_{S}\right)
$$

where $s=\lfloor S\rfloor_{0}, s^{\prime}=\left\lfloor S^{\prime}\right\rfloor_{0}$, and $\Theta(x)$ is 1 for $x>0$ and 0 otherwise.

We should note that $S(t+1)$, given by Eq. (12), is different from $S(t)$ in general even when the player keeps the current strategy $[s(t+1)=s(t)]$. The transition probability $q_{S \rightarrow \tilde{S}}$ from $S$ at $t$ to $\tilde{S}$ at $t+1$ can be written as the sum of two terms,

$$
q_{S \rightarrow \tilde{S}}=\left[w_{S}\left(1-\delta_{s \tilde{s}}\right)+\left(1-w_{S}\right) \delta_{s \tilde{s}}\right] \Lambda_{S \tilde{S}},
$$

where $s=\lfloor S\rfloor_{0}$ and $\tilde{s}=\lfloor\tilde{S}\rfloor_{0}$. The first term represents the transition probability of the player's changing the current strategy $[\tilde{s}=s(t+1) \neq s(t)=s]$; the second term, that of the player's keeping the current strategy $[s(t+1)=s(t)]$.

Generalization for the case with nonzero mutation probability is straightforward. In the presence of a mutation $(\mu>0)$, a player in state $S$ changes his or her current strategy with probability $w_{S}(\mu)$,

$$
w_{S}(\mu)=(1-\mu) w_{S}+\mu\left(1-w_{S}\right),
$$

with $w_{S}$ given by Eq. (15).

We are now ready to write our main equation for the timeevolution of the frequency,

$$
x_{S}(t+1)=x_{S}(t)+\sum_{\tilde{S}}\left[x_{\tilde{S}}(t) q_{\tilde{S} \rightarrow S}-x_{S}(t) q_{S \rightarrow \tilde{S}}\right],
$$

where the transition probability is given by

$$
q_{S \rightarrow \tilde{S}}=\left\{w_{S}(\mu)\left(1-\delta_{s \tilde{s}}\right)+\left[1-w_{S}(\mu)\right] \delta_{s \tilde{S}}\right\} \Lambda_{S \tilde{S}} .
$$

For a given set of our control parameters, $\tau, \eta, \epsilon, c$, and $\mu$, and a given initial goodie frequency, $x_{\mathrm{G}}^{0}$ (with the initial cheater frequency $x_{\mathrm{H}}^{0}=1-x_{\mathrm{G}}^{0}$ ), we numerically iterate the time evolution, Eq. (18), to get the stationary solution, $\mathbf{x}^{\infty}=$ $\mathbf{x}(t \rightarrow \infty)$. Goodies at time $t$ must have $s(t)=1\left(\lfloor S\rfloor_{0}=1\right)$ as their last binary digit, and thus the goodie frequency $x_{\mathrm{G}}$ is given by

$$
x_{\mathrm{G}}(t)=\sum_{k=1}^{N / 2} x_{2 k-1}(t) .
$$

By the same token, the cheater frequency $x_{\mathrm{H}}=1-x_{\mathrm{G}}$ is given by

$$
x_{\mathrm{H}}(t)=\sum_{k=1}^{N / 2} x_{2 k-2}(t) .
$$

\section{RESULTS}

We observe the time evolution of the populations from given initial frequencies and find the final states. First, we find that the final frequencies in a stationary state crucially depend on the initial frequencies for $\mu=0$. We also find that $\eta$ dependency of the stationary frequencies is very intriguing. As $\eta$ varies, the final goodie frequency undergoes a series of sharp drops with fractal-like features (see Appendix A). Although the evolutionary dynamics of a mutation-free population reveals
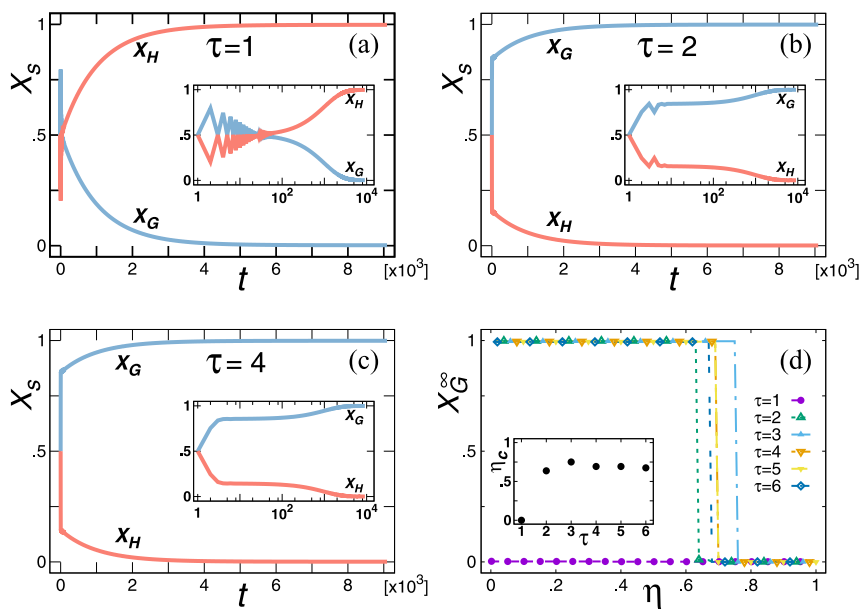

FIG. 1. Time evolution of goodie and cheater frequencies, $x_{\mathrm{G}}$ and $x_{\mathrm{H}}$, for (a) $\tau=1$, (b) $\tau=2$, and (c) $\tau=4$ with $\eta=0.2$. In (a)-(c), we use $c=0.2, \mu=0.001, \epsilon=0$, and $x_{\mathrm{G}}^{0}=0.5$. Populations evolve quite differently for (a) $\tau=1$ and (b, c) $\tau \geqslant 2$. After a sufficiently long time, cheaters dominate for $\tau=1$, while goodies dominate for $\tau \geqslant 2$. Insets in (a)-(c): To clearly show the initial stage of time evolution, we present $x_{\mathrm{G}}$ and $x_{\mathrm{H}}$ in semilog plots here. (d) Stationary goodie frequencies $x_{\mathrm{G}}^{\infty}$ are plotted versus $\eta$ for various $\tau$. The $x_{\mathrm{G}}^{\infty} \approx 1$ for $\eta<\eta_{c}$ and $x_{\mathrm{G}}^{\infty} \approx 0$ otherwise, exhibiting sharp transitions at finite values of $\eta_{c}$ for $\tau \geqslant 2$. However, $x_{\mathrm{G}}^{\infty}=0$ for the whole range of $\eta$ for $\tau=1$. Inset: $\eta_{c}$ vs $\tau$. For $\tau=1, \eta_{c}$ is 0 , but for $\tau \geqslant 2, \eta_{c}$ is around 0.7 .

rich, nontrivial behaviors, we present them in Appendix A, since most of them are not generic phenomena in the sense that they disappear when we turn on mutation.

When we turn on mutation, evolutionary dynamics and the final stationary frequencies are different from the results for $\mu=0$. The final population structure becomes independent of the initial frequencies in many cases. Especially, we find that the population always evolves to a cheater society for $\tau=1$ if goodies have no generosity $(\epsilon=0)$. In the following, we first consider evolutionary dynamics without generosity and then show how generosity can dramatically change the dynamics for $\tau=1$ where forgiveness does not appear.

\section{A. Absence of generosity $(\epsilon=0)$}

We show the time evolution of the goodie and cheater frequencies, $x_{\mathrm{G}}$ and $x_{\mathrm{H}}$, respectively, for $\tau=1$ [Fig. 1(a)], $\tau=2$ [Fig. 1(b)], and $\tau=4$ [Fig. 1(c)], with a small but nonzero mutation probability, $\mu=0.001$. Both frequencies are calculated from the state frequencies $x_{S}(t)$, which we obtain using Eq. (18). After a few thousand transient iterations, the frequencies reach their stationary values. For $\tau=1$, cheaters become dominant regardless of $\eta$ and $x_{\mathrm{G}}^{0}$. However, a cooperative society of goodies can emerge for a finite range of $\eta$ if the memory horizon $\tau$ becomes larger than unity. Before analyzing the evolution of cooperation for $\tau \geqslant 2$, let us first take a look why the goodies always die out for $\tau=1$ in the presence of mutation.

The gruesome future for $\tau=1$ (it may not hold in the structured population in reality, though) can be simply explained as follows (see Fig. 2): In the presence of a mutation, 


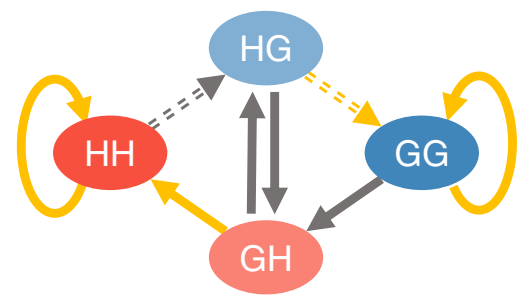

FIG. 2. Schematic of transitions between states with $\tau=1$ in the presence of a mutation with $\epsilon=0$. Gray arrows indicate transitions that occur when the individual changes his or her last strategy; yellow arrows, itransitions when the individual keeps his or her last strategy. Dotted lines denote transitions which occur only through mutations. Where transitions are possible by learning the partner's (higher payoff) strategy, they are represented by thick solid lines. Once GG becomes GH, it easily becomes $\mathrm{HH}$, while a mutant HG from $\mathrm{HH}$ likely returns to $\mathrm{HH}$.

HG can be obtained from $\mathrm{HH}$ through random mutations. When this happens, HG does not play the game with $\mathrm{HH}$ or with $\mathrm{HG}$, since both have zero reputation value. HG may suggest playing to GG, but GG rejects the proposal because of the bad reputation of HG. Accordingly, HG can play the game with GH only. When HG plays the game with $\mathrm{GH}, \mathrm{HG}$ always gets a lower payoff than $\mathrm{GH}$ and, thus, returns to the cheater strategy and evolves to GH. At the next time step, GH becomes either HH (remains a cheater) or HG (changes to a goodie). For the latter, the evolution of $\mathrm{HG}$ is just the one we described above. This process applies iteratively until HG becomes $\mathrm{HH}$. Therefore, HG eventually becomes HH unless unlikely multiple mutations occur in the process. Consequently, state $\mathrm{HH}$ is stable.

Now, let us consider the stability of state GG. It may evolve to GH by higher-payoff-driven "learning" as well as by a pure random mutation. Once this happens, GH eventually evolves to state $\mathrm{HH}$ since $\mathrm{GH}$ is the state in the process in which $\mathrm{HG}$ evolves to HH (see Fig. 2 ). Thus the persistent goodie strategy (characterized by state GG) is not stable, unlike the persistent cheater strategy (characterized by state $\mathrm{HH}$ ). Cooperation in our PDG setting is extremely fragile at $\tau=1$.

One crucial element for the evolution of cheaters is that a repenter HG can play a game with GH only. Unless a mutation occurs, HG returns to the cheater society by learning because GH always gets a higher payoff than HG. Since a repenter goodie HG becomes a cheater at the next time step, HG has no chance of becoming GG.

We may escape from this cheater-dominant situation by providing routes for repenters to join the persistent goodie society. There are at least two ways to do this. We can increase the reputation value of repenters by extending the memory horizon or we can introduce a finite generosity. If we increase the memory horizon to $\tau=2$, there are eight possible states, HHH, HHG, ..., GGG. Now, the repenter HHG at $\tau=2$ becomes HGH first because he or she cannot play a game with goodies due to his or her bad reputation as before. In the next step, however, the repenter can be GHG or GHH. The evolution of GHH is similar to that of $\mathrm{GH}$ for $\tau=1$ : GHH becomes either the persistent cheater state HHH or the repenter HHG. On the other hand, the evolution of GHG is different. It can be HGG
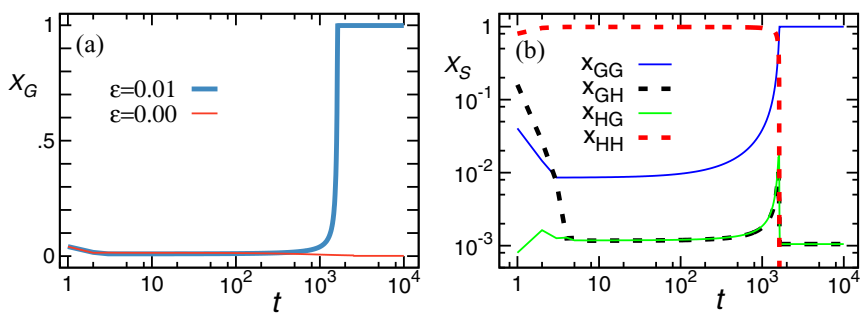

FIG. 3. (a) Evolution of goodies for $\tau=1$ from the initial goodie frequency, $x_{\mathrm{G}}^{0}=0.2$. The time evolution of $x_{\mathrm{G}}$ with $\epsilon=0.01$ is shown by the thick blue line. For comparison, $x_{\mathrm{G}}$ with $\epsilon=0$ is also shown by the thin red line. (b) The frequencies of four states, $x_{\mathrm{HH}}, x_{\mathrm{HG}}, x_{\mathrm{GH}}$, and $x_{\mathrm{GG}}$, in the population in (a) are plotted versus time on a log-log scale. At $t=1, x_{\mathrm{HH}}$ becomes almost 1 and stays there until $t_{q} \approx 10^{3}$ (quasisteady state). Then it drops to its stationary value, $10^{-6}$ (out of plot range), while $x_{\mathrm{GG}}$ soars to its stationary value around 1 . Here, $c=0.2, \eta=0.5$, and $\mu=0.001$ are used.

by learning since GHG can play with goodies. Once a repenter becomes HGG, he or she can join the persistent goodie group of GGG by learning. Hence, for $\tau \geqslant 2$, there is a way for cheaters to escape and join the goodie society. For $\epsilon>0$ with $\tau=1$, evolution of persistent goodies is also possible, since the probability that goodies propose games to repenters is not strictly 0 anymore. We analyze the evolutionary dynamics for $\epsilon>0$ in detail in the following subsection.

\section{B. Effect of generosity $(\epsilon>0)$}

In the previous subsection, we have shown that cooperation cannot be evolved for $\tau=1$ without generosity. We have also observed that the goodies fail to build their stronghold to further spread cooperation across the society, since they turn their faces from newly born goodies. In this subsection, we show how the existence of a small generosity can dramatically change the society. In Fig. 3(a), we present how the goodie frequency $x_{\mathrm{G}}$ evolves with time for $\epsilon=0.01$. For comparison, $x_{\mathrm{G}}$ for $\epsilon=0$ is also presented, as the thin red line. Here, the cheater's game proposal probability is $\eta=0.5$, and the mutation probability is $\mu=0.001$. The evolution of population starts from an initial goodie frequency $x_{\mathrm{G}}^{0}=0.2$. As expected, a small generosity $(\epsilon=0.01)$ does not change the initial dynamics much from the results without generosity $(\epsilon=0)$. However, the results in the stationary state at sufficiently long times are totally different. With generosity, the goodies prevail in the population, while goodies never dominate for $\epsilon=0$.

To understand why a small generosity can change the final goodie frequency so dramatically, we take a closer look at the time evolution of each state. Even for nonzero $\epsilon$, the initial goodie frequency rapidly decreases as long as $\epsilon$ is small, because most HG and $\mathrm{GH}$ become $\mathrm{HH}$ following the process presented for the $\epsilon=0$ case. Then $\mathrm{HG}$ and GH are rare, and the population falls into a quasisteady state in which the population consists mainly of two groups: the major cheater group and minor goodie group. Now interactions between individuals mostly occur within groups, and hence most players in both groups remain in persistent state HH or GG. Figure 3(b) shows the time evolution of the frequencies of four states: $x_{\mathrm{HH}}, x_{\mathrm{HG}}$, $x_{\mathrm{GH}}$, and $x_{\mathrm{GG}}$. Here, we use logarithmic scales for both axes 

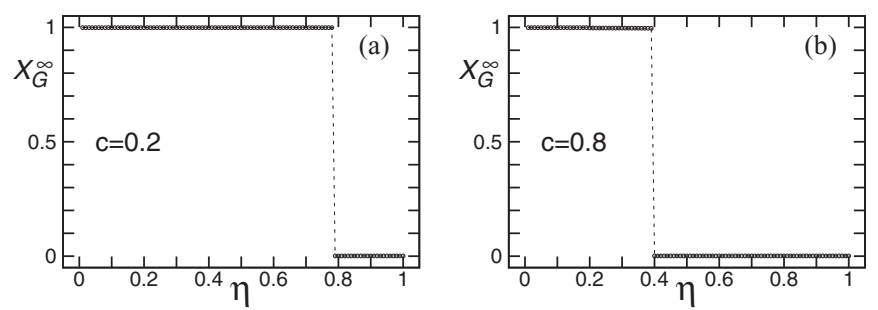

FIG. 4. Stationary-state goodie frequencies for (a) $c=0.2$ and (b) $c=0.8$ with $\epsilon=0.01, \mu=0.001$, and initial frequencies $x_{\mathrm{HH}}=$ $0.499, x_{\mathrm{HG}}=0.001, x_{\mathrm{GH}}=0.001$, and $x_{\mathrm{GG}}=0.499$. Numerical results are consistent with the quasisteady-state approximation, which predicts the evolution of goodies for $\eta<\eta_{c}=0.8$ (a) and $\eta<\eta_{\mathrm{G}} \approx$ 0.4 (b).

to represent changes in low frequencies at early times. After a very short transient time, the population stays in a quasisteady state for a while, up to $t=t_{q} \approx 1000$. Note that both the GG and the $\mathrm{HH}$ groups in the quasisteady state really slowly grow (for $\mathrm{GG}$ ) or shrink (for $\mathrm{HH}$ ) monotonically even though the size of each group looks almost the same. For example, $x_{\mathrm{GG}}$ constantly increases but at a very small increase rate; we can hardly notice its change (unless $t$ approaches $t_{q}$ ) even in the log-scale plot.

To derive the criterion for the growth of the goodie group in the presence of a small generosity, we compare fluxes between two groups in the quasisteady state: fluxes from GG to $\mathrm{HH}$, and vice versa. In the quasisteady state, the frequencies of $\mathrm{HG}$ and $\mathrm{GH}$ are of order $\epsilon$ or $\mu$ as discussed in Appendix B. We calculate the payoffs of four states, $\mathrm{HH}, \mathrm{HG}, \mathrm{GH}$, and GG, up to a linear order in $\epsilon, x_{\mathrm{HG}}$, and $x_{\mathrm{GH}}$. Then the conditions for the evolution of goodies are obtained by considering all possible rank orderings of payoffs of the four states. In Appendix B, we show that goodies proliferate when

$$
\eta<\eta_{t}=\max \left(1-c, \eta_{\mathrm{G}}\right)
$$

with

$$
\eta_{\mathrm{G}}=\frac{\sqrt{x_{\mathrm{GG}}}-x_{\mathrm{GG}}}{1-x_{\mathrm{GG}}},
$$

where $x_{\mathrm{GG}}$ is the frequency of GG in the initial quasisteady state.

For the validity of the quasisteady-state approximation, we calculate the goodie frequency $x_{\mathrm{G}}^{\infty}$ in the stationary state numerically for various values of $\eta$ and plot $x_{\mathrm{G}}^{\infty}$ versus $\eta$ in Fig. 4 for two values of costs, $c=0.2$ and $c=0.8$. Here, our numerical iteration starts with a particular set of frequencies of four states, $x_{\mathrm{HH}}=0.499, x_{\mathrm{HG}}=0.001$, $x_{\mathrm{GH}}=0.001$, and $x_{\mathrm{GG}}=0.499$, for both values of costs. In Fig. 4, we see that the stationary frequencies of goodies $x_{\mathrm{G}}^{\infty}$ are around 1 for $\eta<\eta_{t}$ and 0 otherwise where $\eta_{t} \approx$ 0.8 for $c=0.2$ and $\eta_{t} \approx 0.4$ for $c=0.8$. These results agree very well with the quasisteady-state approximation of Eq. (22). We have $\max \left(1-c, \eta_{\mathrm{G}}\right)=1-c=0.8$ for $c=0.2$ and $\max \left(1-c, \eta_{\mathrm{G}}\right)=\eta_{\mathrm{G}}=\frac{\sqrt{0.499}-0.499}{1-0.499} \approx 0.4$ for $c=0.8$ with $x_{\mathrm{GG}}=0.499$.

Here, we have to mention that it is not always possible to obtain the transition point $\eta_{t}$ analytically from the initial frequency of goodies $x_{\mathrm{G}}^{0}$ since we do not have a formula to
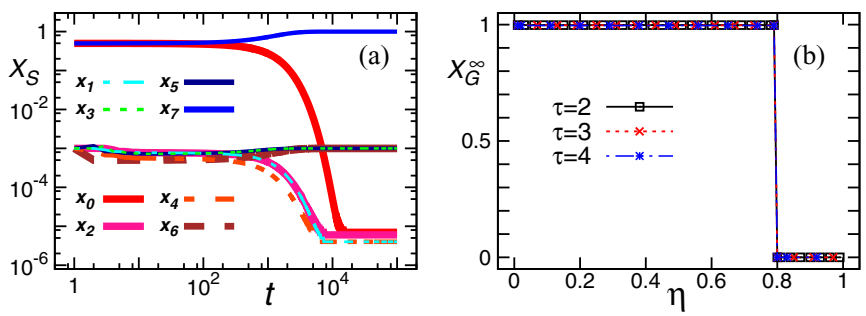

FIG. 5. (a) Evolution of frequencies of eight states for $\tau=2$ and $\eta=0.5$. (b) Goodie frequencies in the stationary state versus $\eta$ for $\tau=2,3$, and 4 with zero generosity and a finite mutation probability. The subscript $S$ in (a) is the decimal number representation of Eq. (6); i.e., $S=0,1, \ldots, 7$ for $\mathrm{HHH}, \mathrm{HHG}, \ldots, \mathrm{GGG}$, respectively. Initial frequencies are given as $x_{S}=\frac{1}{2}[1-\delta(N-2)]$ for persistent states of $S=0$ and $S=N-1$ and as $x_{S}=\delta=0.001$ otherwise. For all cases, $c=0.2, \epsilon=0$, and $\mu=0.001$ are used.

calculate $x_{\mathrm{GG}}$ from $x_{\mathrm{G}}^{0}$. However, $x_{\mathrm{GG}}$ in the initial quasisteady state is obtained numerically very quickly (compared to $x_{\mathrm{G}}^{\infty}$ ), and hence one can well predict the final state from Eq. (22). Furthermore, we can predict the final stationary state by just comparing $\eta$ and $\eta_{c}=1-c$ for $c<1 / 2$, since $\eta_{\mathrm{G}}$ cannot be larger than $1 / 2$. If there is no cost $g$ of playing games, cheaters always dominate by choosing $\eta=1$. In reality, however, it often occurs that playing games requires some cost in general. Because of the risk of losing the game cost $g$, cheaters may reduce their game participation probability $\eta$. We discuss in Appendix B how goodies can evolve when $\eta=1$ in the presence of game cost $g$.

At the end of Sec. IV A, we discussed the emergence of cooperation without an explicit generosity when the memory horizon $\tau$ becomes longer than 1 even if $\epsilon=0$. In Fig. 5(a), we present the evolution of the frequencies of eight states, $\mathrm{HHH}, \mathrm{HHG}, \ldots, \mathrm{GGG}$, of $\tau=2$ with $\epsilon=0$ explicitly. We use $c=0.2, \eta=0.5$, and $x_{S}=0.497$ for two persistent states, $S=$ HHH and $S=$ GGG, and $x_{S}=0.001$ otherwise. The subscript $S$ to $x_{S}$ in Fig. 5(a) is the binary number representation of Eq. (6); i.e., $S=0,1, \ldots, 7$ for $\mathrm{HHH}, \mathrm{HHG}, \ldots, \mathrm{GGG}$, respectively. We present goodie frequencies $x_{\mathrm{G}}=\sum_{k} x_{2 k-1}$ in the stationary states in Fig. 5(b) for $\tau=2$, 3, and 4, with the initial frequencies given by $x_{S}=\frac{1}{2}[1-\Delta(N-2)]$ for persistent states of $S=0$ and $S=N-1$ and by $x_{S}=\Delta=$ 0.001 otherwise. As long as $\tau$ is larger than 1 , the final goodie frequencies seem to be quite independent of $\tau$. As shown in Fig. 5(b), for $c=0.2$ the final goodie frequencies are close to 1 for $\eta<0.8$ and 0 for $\eta>0.8$, similarly to the case of $\tau=1$ with $\epsilon>0$. The condition for the evolution of goodies for $\tau>1$ (with $\epsilon=0$ ) is similar to that for $\tau=1$ (with $\epsilon>0$ ) because the underlying mechanism for the growth of goodie communities is the same. The roles of a long memory horizon $(\tau>1)$ and explicit generosity $(\epsilon>0)$ are the same in the sense that they provide opportunities for repenters to play games with goodies.

\section{DISCUSSION AND SUMMARY}

Generosity and forgiveness together with niceness and provocability have been named as essential ingredients for the evolution of cooperation through direct reciprocity $[9,18]$. 
Here, we show that they are also vital elements to promote cooperation in the optional PDG based on the partner's reputation, where we define reputation as the fraction of cooperation in the past of memory horizon $\tau$. For $\tau=1$, we need generosity for proliferation of goodies. A strict goodie strategy $(\epsilon=0)$ cannot spread because the repentant persons are strictly excluded by the goodies. An $\mathrm{HH}$ player may regret his or her past and become a cooperator HG. However, $\mathrm{HG}$ is already labeled by the past and any goodie refuses to play the game with $\mathrm{HG}$, giving $\mathrm{HG}$ no chance to be a member of a cooperating society. On the other hand, generous goodies $(\epsilon>0)$ provide the chance to keep the repenter's goodie strategy, which is the key mechanism to enhance the cooperative behavior in the society for $\tau=1$. In other words, a nonzero $\epsilon$ allows the new interaction between goodies and repenters with zero reputation, and this interaction can make the society cooperative.

For $\tau \geqslant 2$, a cooperative society can be promoted by forgiveness. In game theory, forgiveness indicates nonzero cooperation probability for the partner in return for cooperation of the opponent, while generosity usually means cooperation with a finite probability even when the partner defects $[18,19,21]$. For $\tau=1$, the reputation value of a repenter is 0 , and the route for forgiveness is blocked. Hence, explicit generosity is needed for the repenter to play with goodies. On the other hand, for $\tau \geqslant 2$, the reputation value of a repenter $(S=1)$ is also 0 , and thus goodies do not play with newborn goodies at first. In that sense, there is no spontaneous forgiveness. However, goodies are forgiving at the next step, remembering the repenter's cooperative behavior in the previous step, and propose games. This provides an opportunity for the repenter to learn about the success of the persistent goodie group.

The roles of generosity of $\epsilon>0$ and forgiveness of $\tau \geqslant 2$ can be unified in the sense that they provide a chance for repenters to play with goodies and make them encounter the better world of cooperation, encouraging them to escape from their isolated world of selfishness. It is well known that both generosity and forgiveness are necessary to achieve a successful cooperative strategy in the iterative PDG [18-22]. On the contrary, just one of them is needed for the evolution of cooperation in our model. Further studies are needed to verify whether this is a generic feature of indirect reciprocity based on reputation.

\section{ACKNOWLEDGMENTS}

H.-C.J. (B.J.K.) was supported by the Basic Science Research Program through the National Research Foundation of Korea funded by the Ministry of Science, ICT, and Future Planning under Grant No. 2015R1D1A1A01058317 (2014R1A2A2A01004919).

\section{APPENDIX A: EVOLUTION WITHOUT MUTATION}

We consider evolutionary dynamics without mutation $(\mu=$ 0 ) for $\tau=1$ and $\epsilon=0$. We vary the cheater's game proposal probability $\eta$ and see how $\eta$ affects the goodie frequency $x_{\mathrm{G}}^{\infty}$ in the stationary state. Figure 6 shows the time evolution of the frequencies of goodies $x_{\mathrm{G}}$ and cheaters $x_{\mathrm{H}}$, from equal goodie
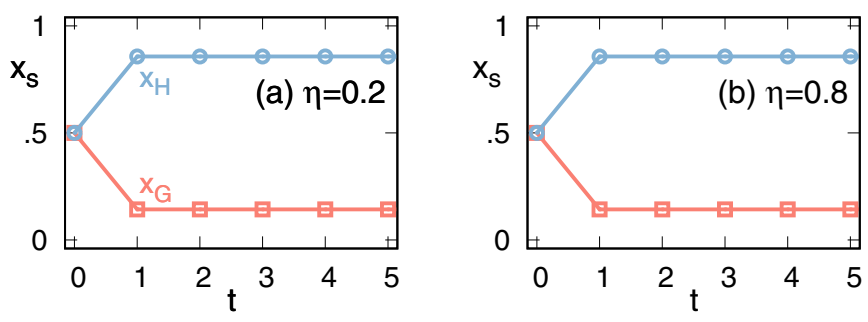

FIG. 6. Frequencies of goodies and cheaters $\left(x_{\mathrm{G}}\right.$ and $\left.x_{\mathrm{H}}\right)$ versus time $t$ for $\tau=1$ with the initial goodie frequency $x_{\mathrm{G}}^{0}=0.5$. Blue circles and red squares represent $x_{\mathrm{G}}$ and $x_{\mathrm{H}}$, respectively. (a) For $\eta=0.2$, goodies eventually flourish $\left(x_{\mathrm{G}}>x_{\mathrm{H}}\right)$, while (b) for $\eta=0.8$, cheaters dominate the system after a transient time. Here, we have used $c=0.2, \mu=0$, and $\epsilon=0$.

and cheater frequencies at $t=0\left(x_{\mathrm{G}}^{0}=x_{\mathrm{H}}^{0}=1 / 2\right)$. For small $\eta$ [for example, $\eta=0.2$ shown in Fig. 6(a)], cheaters have less chance of playing than goodies, even at the beginning. Hence there are only a few newborn cheaters who have a good reputation. Since goodies exclude bad-reputation players, goodies and cheaters segregate into different noninteracting communities after a short transient time and peacefully live on to eternity without making any interaction between the two groups.

For large $\eta$, on the contrary, cheaters eventually flourish instead: At the initial stage of the PDG, cheaters' reputation values do not reflect their true selfish identities, and goodies do not have enough time to realize whom they are facing. The large value of $\eta$ makes it possible that cheaters can play the game with many goodies, and cheaters get high payoffs from such profitable encounters. Several goodies then become envious and turn to cheaters. At the next time step, the remaining goodies play the game with those new cheaters who still have good reputations. This avalanche process stops only when the world is already dominated by cheaters, and goodies can never recover their initial frequency.

For $\mu=0$, the final population structure strongly depends on the initial goodie frequency $x_{\mathrm{G}}^{0}$ as well as the cheater's game proposal probability $\eta$. In Fig. 7(a), we show the goodie frequencies $x_{\mathrm{G}}^{\infty}$ in the stationary state versus $\eta$ for three goodie frequencies $x_{\mathrm{G}}^{0}=0.3,0.5$, and 0.7 . One notable feature is that there are series of sharp drops in the goodie frequencies. At $\eta=\eta_{c_{1}} \approx 1 / 3$ for $x_{\mathrm{G}}^{0}=0.5$, for example, $x_{\mathrm{G}}^{\infty}$ drops below
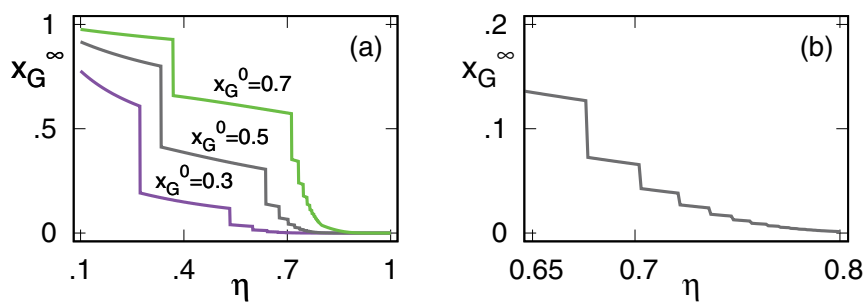

FIG. 7. (a) Goodie frequency $x_{\mathrm{G}}^{\infty}$ in the stationary state for $c=$ $0.2, \epsilon=0$, and $\mu=0$ for three different initial goodie frequencies, $x_{\mathrm{G}}^{0}=0.3,0.5$, and 0.7 . The final goodie frequency $x_{\mathrm{G}}^{\infty}$ undergoes a series of sharp drops as $\eta$ is varied. (b) Magnification of (a) for $x_{\mathrm{G}}^{0}=0.5$ showing its second and subsequent drops. 
half, which is its initial frequency. For $\eta<\eta_{c_{1}}$, the goodies get more payoff than the cheaters $\left(E_{\mathrm{G}}>E_{\mathrm{H}}\right)$ from the beginning. This makes goodies keep their strategies and prohibits newborn cheaters. Then goodies no longer play games with cheaters since all the cheaters are persistent cheaters whose reputation values are 0 . Now the frequency of goodies does not change and this stationary goodie density is higher than $x_{\mathrm{G}}^{0}$ since $E_{\mathrm{G}}>E_{\mathrm{H}}$ from the beginning.

On the other hand, the cheaters defeat the goodies for $\eta>\eta_{c_{1}}$ at the beginning: At $t=1$ a goodie may play the game with a cheater who was a goodie at $t=0$, since this cheater has a fine reputation due to his or her past behavior as a goodie at $t=0$. Such an encounter between a goodie and a new cheater causes the goodie to adopt the cheater strategy, making it a new cheater at $t=2$. Accordingly, for a sufficiently large $\eta$, it is possible that the whole population will soon be occupied by cheaters, resulting in a fast decrease in the goodie frequency.

We can estimate the value of $\eta_{c_{1}}$ from the condition $E_{\mathrm{G}}=E_{\mathrm{H}}$ for a given initial goodie frequency $x_{\mathrm{G}}^{0}$. A goodie cannot distinguish the true identity of his or her game partner at $t=0$ because all agents, goodies and cheaters, have the same reputation value, $r_{0}=1 / 2$, at $t=0$. The goodies propose the game for all other players at a probability $r_{0}=1 / 2$. Cheaters, on the other hand, propose the game with probability $\eta$. Goodies meet other goodies at probability $x_{\mathrm{G}}^{0}$ and play the game with probability $r_{0}^{2}$ since a mutual proposal is needed to play. From such a goodie-goodie encounter, the goodie gets the payoff $b-c$. Accordingly, the goodie's average payoff from other goodies is given by $r_{0}^{2}(b-c) x_{\mathrm{G}}^{0}$. On the other hand, the goodie meets cheaters at probability $1-x_{\mathrm{G}}^{0}$, plays the game at probability $r_{0} \eta$ (again, both must propose), and gets average payoff $-\eta r_{0} c\left(x_{\mathrm{G}}^{0}-1\right)$ from cheaters. Overall, the total payoff $E_{\mathrm{G}}$ of a goodie is written as

$$
E_{\mathrm{G}}=r_{0}^{2}(b-c) x_{\mathrm{G}}^{0}-\eta r_{0} c\left(1-x_{\mathrm{G}}^{0}\right) \text {. }
$$

Likewise, the total payoff $E_{\mathrm{H}}$ of a cheater is computed to yield

$$
E_{\mathrm{H}}=\eta r_{0} b x_{\mathrm{G}}^{0}
$$

From the condition $E_{\mathrm{G}}=E_{\mathrm{H}}$, we get the expression for $\eta_{c_{1}}$ :

$$
\eta_{c_{1}}=\frac{r_{0}(b-c) x_{\mathrm{G}}^{0}}{c+(b-c) x_{\mathrm{G}}^{0}} .
$$

We numerically calculate goodie frequencies at steady state for various values of initial goodie frequencies $x_{\mathrm{G}}^{0}$ and confirm that the first drops in goodie frequencies actually happen at $\eta_{c_{1}}$ given by Eq. (A3). We can also understand the existences of later sudden drops in the goodie frequency in Fig. 7 similarly: Agents with $S=$ GG play the game only with GG and GH because the partner must have a good reputation to play when $\epsilon=0$. If the total payoff of $\mathrm{GG}$ is higher (lower) than that of $\mathrm{GH}$ at $t, \mathrm{H}(\mathrm{G})$ changes to $\mathrm{G}(\mathrm{H})$ at $t+1$. Consequently, the $n$th transition point at $\eta_{c_{n}}$ is determined from the condition $E_{\mathrm{GG}}=E_{\mathrm{GH}}$ at $t=n$, leading to the stepwise transitions shown in Fig. 7. It is interesting that the step structure repeatedly appears ad infinitum at smaller scales as $\eta$ is increased.

\section{APPENDIX B: CRITERION FOR EVOLUTION OF GOODIES: QUASISTEADY-STATE APPROXIMATION}

\section{Quasisteady state}

Here, we study the population dynamics for $\tau=1$ with small $\epsilon$ in the limit of zero mutation. Frequencies and payoffs are calculated up to a linear order in $\epsilon$ and $\mu$ and we consider the limit that $\mu$ goes to 0 before $\epsilon$ does. For small $\epsilon$ and $\mu$, our system quickly falls into a quasisteady state in which almost all members belong to either the persistent goodie (GG) group or the persistent cheater $(\mathrm{HH})$ group, and both groups retain almost-constant sizes. $\mathrm{GH}$ and $\mathrm{HG}$ groups are not completely empty, at least due to the mutation from GG or $\mathrm{HH}$, but their frequencies are of order of $\epsilon$ and $\mu$. Note that GH and HG cannot stay in their own groups. GH must be either $\mathrm{HG}$ or $\mathrm{HH}$ at the next step. Similarly, HG must change to GG or GH in the next step. By the same token, GH and HG cannot come from their own groups. For example, GH must have been GG or HG in the previous step. For GG to be GH by learning, GG should have played with $\mathrm{HH}$ or $\mathrm{GH}$. This happens with probability $\epsilon$ only. GG can be GH by mutation also, which happens with probability $\mu$. Hence, the frequency $x_{\mathrm{GH}}$ of GH is of order $\epsilon$ or $\mu$ when $x_{\mathrm{GG}}$ and $x_{\mathrm{HH}}$ are of order 1. In parallel, $x_{\mathrm{HG}}$ is also of order $\epsilon$ or $\mu$. We calculate below the payoffs of four states, $\mathrm{HH}, \mathrm{HG}, \mathrm{GH}$, and GG, up to a linear order in $\epsilon, x_{\mathrm{HG}}$, and $x_{\mathrm{GH}}$.

\section{Payoffs in the quasisteady state}

The probability $p_{S S^{\prime}}$ for the mutual proposals between $S$ and $S^{\prime}$ states is given by

$$
\begin{aligned}
p_{S S^{\prime}} & =p_{s}\left(r_{S^{\prime}}\right) p_{s^{\prime}}\left(r_{S}\right) \\
& =\left(\left[(1-\epsilon) r_{S^{\prime}}\right] s+\eta[1-s]\right)\left(\left[(1-\epsilon) r_{S}\right] s^{\prime}+\eta\left[1-s^{\prime}\right]\right),
\end{aligned}
$$

as discussed in the text. For $\tau=1$, we have four possible states, $S=\mathrm{HH}, \mathrm{HG}, \mathrm{GH}$, and $\mathrm{GG}$, and their reputation value $r_{S}$ and current strategy $s(S)$ are given as $r_{S}=0,0,1$, and 1 and $s(S)=0,1,0$, and 1 for $S=\mathrm{HH}, \mathrm{HG}, \mathrm{GH}$, and GG, respectively. The mutual proposal probability $p_{S S^{\prime}}$ between two states $S$ and $S^{\prime}$ can be tabulated as follows:

\begin{tabular}{lcccc}
\hline \hline$S$ & \multicolumn{3}{c}{$S^{\prime}$} \\
& $\mathrm{HH}$ & $\mathrm{HG}$ & $\mathrm{GH}$ & $\mathrm{GG}$ \\
\cline { 2 - 4 } & $\eta^{2}$ & $\eta \epsilon$ & $\eta^{2}$ & $\eta \epsilon$ \\
$\mathrm{HH}$ & $\eta \epsilon$ & $\epsilon^{2}$ & $\eta$ & $\epsilon$ \\
$\mathrm{GH}$ & $\eta^{2}$ & $\eta$ & $\eta^{2}$ & $\eta$ \\
$\mathrm{GG}$ & $\epsilon \eta$ & $\epsilon$ & $\eta$ & 1 \\
\hline \hline
\end{tabular}

Now using the payoff matrix of the PDG,

$$
\mathbf{M}=\left(\begin{array}{cc}
b-c & -c \\
b & 0
\end{array}\right),
$$

we can calculate the payoffs for each state up to a linear order in $\epsilon, x_{\mathrm{GH}}$, and $x_{\mathrm{HG}}$ :

$$
\begin{aligned}
E_{\mathrm{HH}} & =\epsilon \eta b\left(x_{\mathrm{HG}}+x_{\mathrm{GG}}\right) \approx \epsilon \eta b x_{\mathrm{GG}}, \\
E_{\mathrm{HG}} & =\epsilon \eta(-c) x_{\mathrm{HH}}+\epsilon^{2}(b-c) x_{\mathrm{HG}}+\eta(-c) x_{\mathrm{GH}}+\epsilon(b-c) x_{\mathrm{GG}} \\
& \approx-\epsilon \eta c x_{\mathrm{HH}}-\eta c x_{\mathrm{GH}}+\epsilon(b-c) x_{\mathrm{GG}},
\end{aligned}
$$




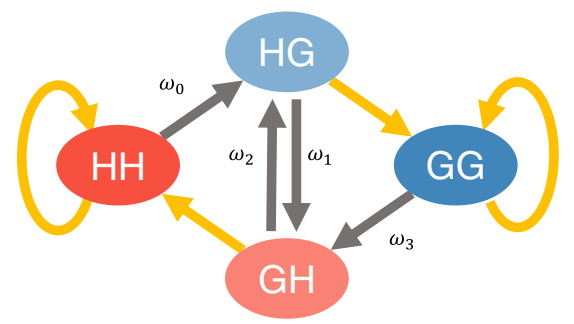

FIG. 8. Possible transitions between two states of unit memory. Yellow arrows indicate the transition when a player keeps his or her last strategy; gray arrows, the transition when the player changes the strategy. Here, $w_{i}$ represents the strategy change probability of state $\mathrm{S}_{i}$.

$$
\begin{aligned}
E_{\mathrm{GH}} & =\eta b\left(x_{\mathrm{HG}}+x_{\mathrm{GG}}\right) \approx \eta b\left(x_{\mathrm{HG}}+x_{\mathrm{GG}}\right), \\
E_{\mathrm{GG}} & =\epsilon \eta(-c) x_{\mathrm{HH}}+\epsilon(b-c) x_{\mathrm{HG}}+\eta(-c) x_{\mathrm{GH}}+(b-c) x_{\mathrm{GG}} \\
& \approx-\epsilon \eta c x_{\mathrm{HH}}-\eta c x_{\mathrm{GH}}+(b-c) x_{\mathrm{GG}} .
\end{aligned}
$$

The population dynamics is mainly determined by the rankings of these four payoffs since the players are supposed to follow the successful strategies of neighbors. We first note that the inequality $E_{\mathrm{HG}}<E_{\mathrm{GG}}$ and $E_{\mathrm{HH}}<E_{\mathrm{GH}}$ always holds since the players with better reputations always earn higher payoff when they have the same current strategy. Furthermore, we restrict our model to $E_{\mathrm{HH}}<E_{\mathrm{GG}}$ and $E_{\mathrm{HG}}<E_{\mathrm{GH}}$ cases only. If $E_{\mathrm{HH}}>E_{\mathrm{GG}}$, goodies never prevail in the population since $\mathrm{G}$ becomes a strictly dominated strategy. On the other hand, $\mathrm{H}$ becomes a strictly dominated strategy if $E_{\mathrm{HG}}>E_{\mathrm{GH}}$. Now, only two cases remain: (i) $E_{\mathrm{GH}}<E_{\mathrm{GG}}$, and (ii) $E_{\mathrm{GG}}<E_{\mathrm{GH}}$. We calculate below the conditions for the evolution of goodies in both cases.

\section{Condition for evolution of goodies}

In the quasisteady state, the population mainly consists of GG and HH groups. The sizes of the two groups are almost constant but change very slowly. In other words, there is small but nonzero flux between two groups. We find the conditions for the evolution of goodies by calculating the net flux between $\mathrm{GG}$ and $\mathrm{HH}$ states. For the evolution of goodies, the influx $F_{\text {in }}$ from $\mathrm{HH}$ to GG should be larger than the outflux $F_{\text {out }}$ from GG to HH. Let us introduce $w_{i}$ to denote the probability that an individual in state $S_{i}$ (with $S_{i}=\mathrm{HH}, \mathrm{HG}, \mathrm{GH}$, and GG for $i=0,1,2$, and 3 , respectively) will change his or her current strategy (see Fig. 8). Here, $w_{i}$ is a function of $x_{\mathrm{GG}}$ and $x_{\mathrm{HH}}$ and hence it is not a constant, but its changing probability is of order $\epsilon$ and $\mu$ only. If we treat $w_{i}$ as a constant, $F_{\text {in }}$ and $F_{\text {out }}$ can be expressed in terms of $w_{i}$ as

$$
\begin{aligned}
F_{\text {in }} & =x_{\mathrm{HH}} w_{0}\left(1-w_{1}\right)\left[1+w_{2} w_{1}+\left(w_{2} w_{1}\right)^{2}+\ldots\right] \\
& =x_{\mathrm{HH}} w_{0} \frac{1-w_{1}}{1-w_{2} w_{1}}, \\
F_{\mathrm{out}} & =x_{\mathrm{GG}} w_{3}\left(1-w_{2}\right)\left[1+w_{1} w_{2}+\left(w_{1} w_{2}\right)^{2}+\ldots\right] \\
& =x_{\mathrm{GG}} w_{3} \frac{1-w_{2}}{1-w_{2} w_{1}} .
\end{aligned}
$$

The condition $F_{\text {out }}<F_{\text {in }}$ for the evolution of goodies is then reduced to

$$
x_{\mathrm{GG}} w_{3}\left(1-w_{2}\right)<x_{\mathrm{HH}} w_{0}\left(1-w_{1}\right)
$$

\section{a. The $E_{\mathrm{GH}}<E_{\mathrm{GG}}$ case}

For $E_{\mathrm{GH}}<E_{\mathrm{GG}}$, we find that the population always evolves to a goodie society once the quasisteady state is established. First, note that $E_{\mathrm{GH}}<E_{\mathrm{GG}}$ implies

$$
\eta<\eta_{c} \equiv(1-c / b)
$$

for small $\epsilon$ and $\mu$, since $E_{\mathrm{GH}} \approx \eta b x_{\mathrm{GG}}$ and $E_{\mathrm{GG}} \approx(b-c) x_{\mathrm{GG}}$ in the zeroth-order approximation. To determine the condition for the evolution of goodies, we then calculate $w_{i}^{0}$, the strategy change probabilities $w_{i}$ for $\mu=0$. When $E_{\mathrm{GH}}<E_{\mathrm{GG}}$, they are given by

$$
\begin{aligned}
w_{0}^{0} & =\frac{\epsilon \eta x_{\mathrm{GG}}}{\epsilon \eta\left(x_{\mathrm{HG}}+x_{\mathrm{GG}}\right)+\eta^{2}\left(x_{\mathrm{HH}}+x_{\mathrm{GH}}\right)} \approx \frac{\epsilon x_{\mathrm{GG}}}{\eta x_{\mathrm{HH}}}, \\
w_{1}^{0} & =\frac{\epsilon \eta x_{\mathrm{HH}}+\eta x_{\mathrm{GH}}}{\epsilon \eta x_{\mathrm{HH}}+\epsilon^{2} x_{\mathrm{HG}}+\eta x_{\mathrm{GH}}+\epsilon x_{\mathrm{GG}}} \\
& \approx \frac{\epsilon \eta x_{\mathrm{HH}}+\eta x_{\mathrm{GH}}}{\epsilon \eta x_{\mathrm{HH}}+\eta x_{\mathrm{GH}}+\epsilon x_{\mathrm{GG}}}, \\
w_{2}^{0} & =\frac{x_{\mathrm{GG}}}{\eta x_{\mathrm{HH}}+x_{\mathrm{HG}}+\eta x_{\mathrm{GH}}+x_{\mathrm{GG}}} \approx \frac{x_{\mathrm{GG}}}{\eta x_{\mathrm{HH}}+x_{\mathrm{GG}}}, \\
w_{3}^{0} & =0 .
\end{aligned}
$$

For $\mu>0$, the $w_{i}$ are given by

$$
w_{i}(\mu)=w_{i}^{0}(1-\mu)+\left(1-w_{i}^{0}\right) \mu .
$$

Since $x_{\mathrm{HG}}$ and $x_{\mathrm{GH}}$ are constant (up to a linear order in $\epsilon$ and $\mu$ ) in the quasisteady state, the influxes to these states should be the same as the outfluxes. From Fig. 8, we have

$$
x_{\mathrm{HG}}=w_{0} x_{\mathrm{HH}}+w_{2} x_{\mathrm{GH}}, \quad x_{\mathrm{GH}}=w_{3} x_{\mathrm{GG}}+w_{1} x_{\mathrm{HG}} .
$$

Solving Eq. (B9) with the strategy change probabilities given by Eq. (B8), we get

$$
\begin{aligned}
& x_{\mathrm{HG}}=\epsilon \frac{x_{\mathrm{GG}}\left(\eta x_{\mathrm{HH}}+2 x_{\mathrm{GG}}\right)}{\eta\left(\eta x_{\mathrm{HH}}+x_{\mathrm{GG}}\right)}+\mu \frac{\eta x_{\mathrm{HH}}^{2}+x_{\mathrm{GG}}\left(x_{\mathrm{HH}}+x_{\mathrm{GG}}\right)}{\eta x_{\mathrm{HH}}}, \\
& x_{\mathrm{GH}}=\epsilon \frac{x_{\mathrm{GG}}}{\eta}+\mu \frac{\left(x_{\mathrm{HH}}+x_{\mathrm{GG}}\right)\left(\eta x_{\mathrm{HH}}+x_{\mathrm{GG}}\right)}{\eta x_{\mathrm{HH}}}
\end{aligned}
$$

Now $w_{i}$ can be expressed in terms of $x_{\mathrm{HH}}$ and $x_{\mathrm{GG}}$ as

$$
\begin{aligned}
w_{0} & =\frac{x_{\mathrm{GG}}}{\eta x_{\mathrm{HH}}} \epsilon+\mu, \\
w_{1} & =\frac{\left(\eta x_{\mathrm{HH}}+x_{\mathrm{GG}}\right) x_{\mathrm{HH}} \epsilon+\left(\eta x_{\mathrm{HH}}+x_{\mathrm{GG}}\right)\left(x_{\mathrm{HH}}+x_{\mathrm{GG}}\right) \mu}{\left(\eta x_{\mathrm{HH}}+2 x_{\mathrm{GG}}\right) x_{\mathrm{HH}} \epsilon+\left(\eta x_{\mathrm{HH}}+x_{\mathrm{GG}}\right)\left(x_{\mathrm{HH}}+x_{\mathrm{GG}}\right) \mu}, \\
w_{2} & =\frac{x_{\mathrm{GG}}}{\eta x_{\mathrm{HH}}+x_{\mathrm{GG}}}, \\
w_{3} & =\mu .
\end{aligned}
$$

In the zero-mutation limits, $w_{3}$ goes to 0 first and the condition $F_{\text {out }}<F_{\text {in }}$ of inequality (B5) always holds. In other words, for $E_{\mathrm{GH}}<E_{\mathrm{GG}}$ (i.e., $\eta<\eta_{c}$ ) the evolution of cooperation is guaranteed. 


\section{b. The $E_{\mathrm{GG}}<E_{\mathrm{GH}}$ case}

We first note that $\eta$ should be larger than $\eta_{c}=1-c / b$ in this case since $E_{\mathrm{GH}} \approx \eta b x_{\mathrm{GG}}$ and $E_{\mathrm{GG}} \approx(b-c) x_{\mathrm{GG}}$ as discussed before. When $\eta>\eta_{c}, E_{\mathrm{HH}}$ is always larger than $E_{\mathrm{HG}}$ and the strategy change probabilities of zero mutation $w_{i}^{0}$ are given by

$$
\begin{aligned}
w_{0}^{0} & =\frac{\epsilon \eta x_{\mathrm{GG}}}{\epsilon \eta\left(x_{\mathrm{HG}}+x_{\mathrm{GG}}\right)+\eta^{2}\left(x_{\mathrm{HH}}+x_{\mathrm{GH}}\right)} \approx \frac{\epsilon x_{\mathrm{GG}}}{\eta x_{\mathrm{HH}}}, \\
w_{1}^{0} & =\frac{\epsilon \eta x_{\mathrm{HH}}+\eta x_{\mathrm{GH}}}{\epsilon \eta x_{\mathrm{HH}}+\epsilon^{2} x_{\mathrm{HG}}+\eta x_{\mathrm{GH}}+\epsilon x_{\mathrm{GG}}} \\
& \approx \frac{\epsilon \eta x_{\mathrm{HH}}+\eta x_{\mathrm{GH}}}{\epsilon \eta x_{\mathrm{HH}}+\eta x_{\mathrm{GH}}+\epsilon x_{\mathrm{GG}}}, \quad w_{2}^{0}=0, \\
w_{3}^{0} & =\frac{\eta x_{\mathrm{GH}}}{\epsilon \eta x_{\mathrm{HH}}+\epsilon x_{\mathrm{HG}}+\eta x_{\mathrm{GH}}+x_{\mathrm{GG}}} \approx \frac{\eta x_{\mathrm{GH}}}{x_{\mathrm{GG}}} .
\end{aligned}
$$

We get $x_{\mathrm{HG}}$ and $x_{\mathrm{GH}}$ in terms of $x_{\mathrm{HH}}$ and $x_{\mathrm{GG}}$ by solving the quasisteady-state condition of Eq. (B9) with $w_{i}$ of Eq. (B8) calculated with $w_{i}^{0}$ of Eq. (B12). They are given by

$x_{\mathrm{HG}}=\epsilon \frac{x_{\mathrm{GG}}}{\eta}+\mu x_{\mathrm{HH}}$,

$x_{\mathrm{GH}}=-\epsilon \frac{x_{\mathrm{HH}}}{2}+\epsilon \frac{\eta x_{\mathrm{GG}}+\sqrt{R\left(x_{\mathrm{HH}}, x_{\mathrm{GG}}, \eta\right)}}{2 \eta(1-\eta)}+\mu \frac{x_{\mathrm{HH}}+x_{\mathrm{GG}}}{1-\eta}$,

where

$R\left(x_{\mathrm{HH}}, x_{\mathrm{GG}}, \eta\right)=4 \eta x_{\mathrm{HH}} x_{\mathrm{GG}}(1-\eta)+\eta^{2}\left[x_{\mathrm{GG}}-x_{\mathrm{HH}}(1-\eta)\right]^{2}$.

Now all $w_{i}$ can be written in terms of $x_{\mathrm{HH}}$ and $x_{\mathrm{GG}}$, and inequality (B5) becomes

$$
x_{\mathrm{GG}}[2-\eta(4-\eta)]>x_{\mathrm{HH}} \eta^{2}(1-\eta)+\eta \sqrt{R}
$$

in the zero-mutation limit. Since the population mainly consists of GG and $\mathrm{HH}$ groups, we apply $x_{\mathrm{GG}}+x_{\mathrm{HH}} \approx 1$ to inequality (B15) and get the condition for the evolution of cooperation for the $E_{\mathrm{GG}}<E_{\mathrm{GH}}$ case. It is simply written as

$$
\eta<\eta_{\mathrm{G}}
$$

with

$$
\eta_{\mathrm{G}}=\frac{\sqrt{x_{\mathrm{GG}}}-x_{\mathrm{GG}}}{1-x_{\mathrm{GG}}} .
$$

Since $E_{\mathrm{GG}}<E_{\mathrm{GH}}$ implies $\eta>\eta_{c}=1-c / b$, the evolution of cooperation occurs when

$$
\eta_{c}<\eta<\eta_{\mathrm{G}}
$$

We would like to make two comments on the above inequality. First, it cannot have a solution if $\eta_{c}>1 / 2$ (equivalently, $c / b<$ $1 / 2$ ) since $\eta_{\mathrm{G}}$ cannot be larger than $1 / 2$. Second, $x_{\mathrm{GG}}$ in $\eta_{\mathrm{G}}$ is the frequency of GG in the initial quasisteady state. It is determined by the initial frequency $x_{\mathrm{G}}^{0}$ but is hard to estimate from $x_{\mathrm{G}}^{0}$ since the population dynamics to the quasisteady state during the transient time is nontrivial.
Together with the case of $E_{\mathrm{GH}}<E_{\mathrm{GG}}$, the condition for evolution of cooperation in the zero-mutation limit can be summarized as

$$
\eta< \begin{cases}1-c / b & \text { for } b / c<1 / 2, \\ \max \left(1-b / c, \eta_{\mathrm{G}}\right) & \text { otherwise, }\end{cases}
$$

where $\eta_{\mathrm{G}}=\frac{\sqrt{x_{\mathrm{GG}}}-x_{\mathrm{GG}}}{1-x_{\mathrm{GG}}}$.

\section{Evolution with the game cost $g$ for $\eta=1$}

If there is no cost for playing games, cheaters may propose games for everyone $(\eta=1)$. In reality, however, playing games often requires paying cost $g$ in general. When we introduce the game cost $g$, the game payoff matrix is written as

$$
\mathbf{M}=\left(\begin{array}{cc}
b-c-g & -c-g \\
b-g & -g
\end{array}\right)
$$

for $0 \leqslant g \leqslant 1-c$. If the number of game opponents is fixed, the game cost $g$ will not play any role. However, due to the refusal option in our model, $g$ represents the benefit of not playing the games. Here, we calculate the evolution condition of goodies when cheaters always participate in games with anyone $(\eta=1)$. In the presence of $g$, the average payoff in Eq. (B3) is written differently, and so is $w_{i}$. We take a closer look at two cases: (i) $E_{\mathrm{GH}}<E_{\mathrm{GG}}$ and (ii) $E_{\mathrm{GH}}>E_{\mathrm{GG}}$.

The first condition, $E_{\mathrm{GH}}<E_{\mathrm{GG}}$, holds when

$$
x_{\mathrm{GG}}>\frac{g}{c+g} \text {. }
$$

Since rankings of four payoffs are changed, $w_{i}$ 's are given by

$$
\begin{aligned}
& w_{0}^{0} \approx \frac{\epsilon x_{\mathrm{GG}}}{x_{\mathrm{HH}}}, \quad w_{1}^{0} \approx \frac{x_{\mathrm{GH}}}{\epsilon x_{\mathrm{HH}}+x_{\mathrm{GH}}+\epsilon x_{\mathrm{GG}}}, \\
& w_{2}^{0} \approx \frac{x_{\mathrm{GG}}}{x_{\mathrm{HH}}+x_{\mathrm{GG}}}, \quad w_{3}^{0}=0 .
\end{aligned}
$$

For new expressions for $w_{i}$, Eqs. (B9) are rewritten as follows:

$$
\begin{aligned}
& x_{\mathrm{HG}} \approx \mu x_{\mathrm{HH}}+\epsilon x_{\mathrm{GG}}+\frac{x_{\mathrm{GH}} x_{\mathrm{GG}}}{x_{\mathrm{HH}}+x_{\mathrm{GG}}}, \\
& x_{\mathrm{GH}} \approx \mu x_{\mathrm{GG}}+\frac{x_{\mathrm{HG}} x_{\mathrm{GH}}}{x_{\mathrm{GH}}+\epsilon\left(x_{\mathrm{GG}}+x_{\mathrm{HH}}\right)} .
\end{aligned}
$$
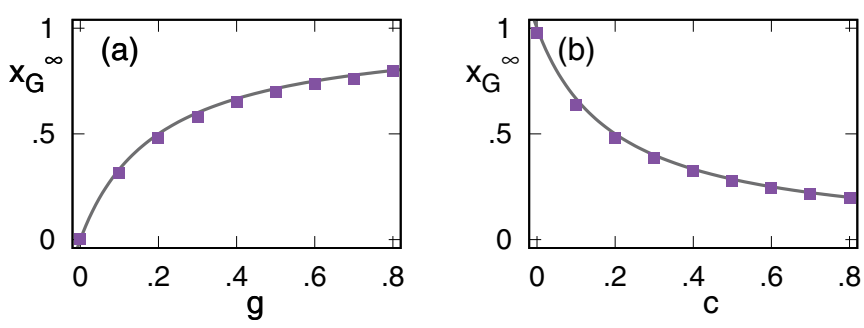

FIG. 9. Goodie frequencies $x_{G}$ (a) versus $g$ at $c=0.2$ and (b) versus $c$ at $g=0.2$. Note that the condition $g+c<b$ must be satisfied. Squares are from simulation results and agree well with the solid gray line $g /(c+g)$ obtained from analytic calculation (see text). We have used the initial goodie frequency $x_{\mathrm{G}}^{0}=0.5$. 
Using $x_{\mathrm{GG}}+x_{\mathrm{HH}} \approx 1$ and keeping the leading order, we get the following solutions:

$$
x_{\mathrm{HG}} \approx \mu \frac{x_{\mathrm{HH}}+x_{\mathrm{GG}}^{2}}{x_{\mathrm{HH}}}+\epsilon x_{\mathrm{GG}}, \quad x_{\mathrm{GH}} \approx \frac{\mu}{x_{\mathrm{HH}}} .
$$

These solutions always satisfy the condition for evolution of goodies in Eq. (B5), and thus goodies always dominate when $x_{\mathrm{GG}}>\frac{g}{c+g}$.

On the other hand, cheaters always prevail for $E_{\mathrm{GG}}<E_{\mathrm{GH}}$ as shown below. We show that goodies cannot take over the entire population when

$$
x_{\mathrm{GG}}<\frac{g}{c+g} \text {. }
$$

In this case, $w_{i}$ 's are written

$$
\begin{aligned}
& w_{0}^{0} \approx \frac{\epsilon x_{\mathrm{GG}}}{x_{\mathrm{HH}}}, \quad w_{1}^{0} \approx \frac{x_{\mathrm{GH}}}{\epsilon x_{\mathrm{HH}}+x_{\mathrm{GH}}+\epsilon x_{\mathrm{GG}}}, \\
& w_{2}^{0}=0, \quad w_{3}^{0} \approx \frac{x_{\mathrm{GH}}}{x_{\mathrm{GG}}} .
\end{aligned}
$$

These new $w_{i}$ 's directly give the solution of $x_{\mathrm{HG}}$ from Eqs. (B9), yielding $x_{\mathrm{HG}} \approx \mu x_{\mathrm{HH}}+\epsilon x_{\mathrm{GG}}$. Because the second Eq. (B9) cannot determine $x_{\mathrm{GH}}$, we get $x_{\mathrm{GH}}$ from the first equation using the solution of $x_{\mathrm{HG}}$. We can get $x_{\mathrm{HG}}=2 \epsilon x_{\mathrm{GG}}$, and these solutions yield the evolution of cheaters. Hence, cheaters dominate when $x_{\mathrm{GG}}<\frac{g}{c+g}$. Combining the two results, we conclude that the goodie frequency remains at $\frac{g}{c+g}$ because there are two opposite flows towards $\frac{g}{c+g}$. The prediction is in good agreement with our simulation results (see Fig. 9).
[1] M. A. Nowak, Evolutionary Dynamics (Harvard University Press, Cambridge, MA, 2006).

[2] J. Maynard Smith, Evolution and the Theory of Games (Cambridge University Press, Cambridge, UK, 1982).

[3] M. A. Nowak, Science 314, 1560 (2006).

[4] G. Szabó and G. Fáth, Phys. Rep. 446, 97 (2007).

[5] R. D. Alexander, The Biology of Moral Systems (Aldine de Gruyter, New York, 1987).

[6] M. A. Nowak and K. Sigmund, Nature 393, 573 (1998).

[7] M. A. Nowak and K. Sigmund, Nature 437, 1291 (2005).

[8] W. Ghang and M. A. Nowak, J. Theor. Biol. 365, 1 (2015).

[9] T. Röhl, C. Röhl, H. G. Schuster, and A. Traulsen, Phys. Rev. E 76, 026114 (2007).

[10] F. Fu, C. Hauert, M. A. Nowak, and L. Wang, Phys. Rev. E 78, 026117 (2008).

[11] Z. Yang, Z. Li, T. Wu, and L. Wang, Phys. Rev. E 88, 042128 (2013).
[12] R. Trivers, Q. Rev. Biol. 46, 35 (1971).

[13] G. E. Bolton, E. Katok, and A. Ockenfels, Manage. Sci. 50, 1587 (2004).

[14] P. Resnick, R. Zeckhauser, E. Friedman, and K. Kuwabara, Commun. ACM 43, 45 (2000).

[15] C. Hauert, S. De Monte, J. Hofbauer, and K. Sigmund, J. Theor. Biol. 218, 187 (2002).

[16] S. S. Izquierdo, L. R. Izquierdo, and F. Vega-Redondo, J. Theor. Biol. 267, 76 (2010).

[17] H.-C. Jeong, S.-Y. Oh, B. Allen, and M. A. Nowak, J. Theor. Biol. 356, 98 (2014).

[18] R. Axelrod, The Evolution of Cooperation, rev. ed. (Basic Books, Cambridge, MA, 2009).

[19] R. Axelrod and D. Dion, Science 242, 1385 (1988).

[20] M. A. Nowak, J. Theor. Biol. 142, 237 (1990).

[21] M. A. Nowak and K. Sigmund, Acta Appl. Math. 20, 247 (1990).

[22] M. A. Nowak and K. Sigmund, Nature 355, 250 (1992). 